\title{
ON THE MESH DIVERGENCE OF INVISCID ADJOINT SOLUTIONS
}

\section{CARLOS LOZANO¹ AND JORGE PONSIN ${ }^{2}$}

\author{
Computational Aerodynamics Group \\ National Institute of Aerospace Technology (INTA). \\ Carretera de Ajalvir, km 4. Torrejón de Ardoz 28850, Spain. \\ 1'lozanorc@inta.es, 2ponsinj@inta.es
}

Key words: Inviscid Adjoint Solution, Mesh Dependence.

\begin{abstract}
Certain two and three dimensional numerical solutions to the adjoint Euler equations have a value at and near the surface of wings and airfoils that depends strongly on the mesh density and which does not converge as the mesh is refined. The purpose of this paper is to characterize this problem and offer insights as to the possible explanation of this unusual behavior.
\end{abstract}

\section{INTRODUCTION}

Recently, it has been shown [1] that in two and three dimensions, certain numerical adjoint solutions to the Euler equations have values at and near the surface of wings and airfoils that depend strongly on the mesh density and which do not converge as the mesh is refined. This phenomenon has been observed for lift-based adjoint solutions for any subcritical or transonic flow condition, while for drag-based adjoint solutions it has only been observed in transonic rotational flows.

The problem seems to be rather generic, as it has been found in solutions obtained with continuous and discrete adjoint schemes and with different solvers. Increasing the numerical dissipation with mesh refinement does not qualitatively change the behavior, although the actual value of the adjoint at the wall strongly depends on the level of numerical dissipation. It was conjectured in [1] that this behavior is likely caused by the adjoint singularity at the sharp trailing edge, although an understanding of the actual mechanism was lacking.

Here, we offer some new insights concerning this problem. We analyze its dependence on the far-field distance and resolution, and also explore a different cost function measuring farfield entropy flux, which shows the same behavior as the near-field drag. Finally, we show that the mesh-divergence is also correlated to the adjoint singularity along the incoming stagnation streamline [2], and it appears also in flows past blunt bodies without sharp trailing edges. Finally, numerical experiments with point sources hint at a singular behavior at the wall, the nature of which is briefly discussed. 


\section{CHARACTERIZATION OF THE PROBLEM}

To introduce the problem, we examine a fairly simple example: the drag-based adjoint solution for inviscid transonic flow past a NACA0012 airfoil with sharp trailing edge at Mach number $M_{\infty}=0.8$ and angle of attack $\alpha=1.25^{\circ}$. The solution obeys the Euler adjoint equation $\nabla \psi^{T} \cdot \vec{F}_{U}=0$ with adjoint wall boundary condition $\left(\psi_{x}, \psi_{y}\right) \cdot \vec{n}_{S}=\vec{d} \cdot \vec{n}_{S}$ and dual characteristic b.c. $\psi^{T}\left(\vec{F}_{U} \cdot \vec{n}_{S^{\infty}}\right) \delta U=0$ at the far-field. Here $\psi^{T}=\left(\psi_{1}, \psi_{x}, \psi_{y}, \psi_{4}\right)$ is the adjoint state, $\vec{F}_{U}$ is the flux Jacobian, $\delta U$ is the linear flow perturbation, $\vec{n}_{S}$ is the wall normal vector and $\vec{d}=(\cos \alpha, \sin \alpha) \quad(\vec{d}=(-\sin \alpha, \cos \alpha)$ for lift $)$. This case should be straightforward to solve numerically, but turns out to yield unexpected results. Plotting the adjoint values on the airfoil profile across several mesh levels, one would expect to see a singularity at the trailing edge [3] and also at the shock and the entrance to the supersonic zone, with the solution along the remainder of the profile remaining stable or progressively converging over successive mesh levels. This is not what is observed: instead, the numerical solution diverges across the entire airfoil profile as the mesh density increases (Figure 1-left)

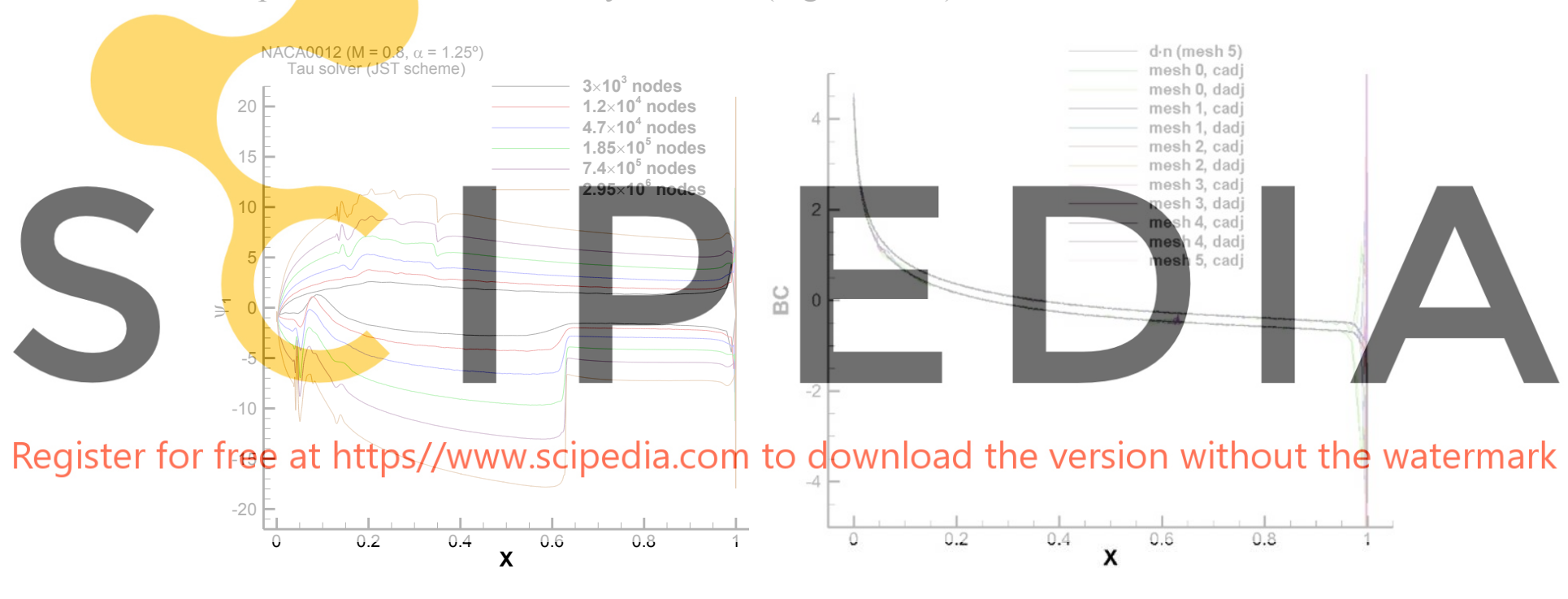

Figure 1. Drag adjoint solution on a NACA0012 airfoil at $M_{\infty}=0.8, \alpha=1.25^{\circ}$ computed with DLR's Tau code [4] on 6 progressively refined meshes. Left: $\psi_{1}$ on the airfoil. Right: Numerical vs. analytic wall adjoint b.c.

The wall b.c. $\left(\psi_{x}, \psi_{y}\right) \cdot \vec{n}_{S}=\vec{d} \cdot \vec{n}_{S}$ is reasonably well obeyed across mesh levels except in the immediate vicinity of the trailing edge (Figure 1-right). Likewise, sensitivity derivatives are actually quite accurate and fairly stable across mesh levels (Figure 2). 

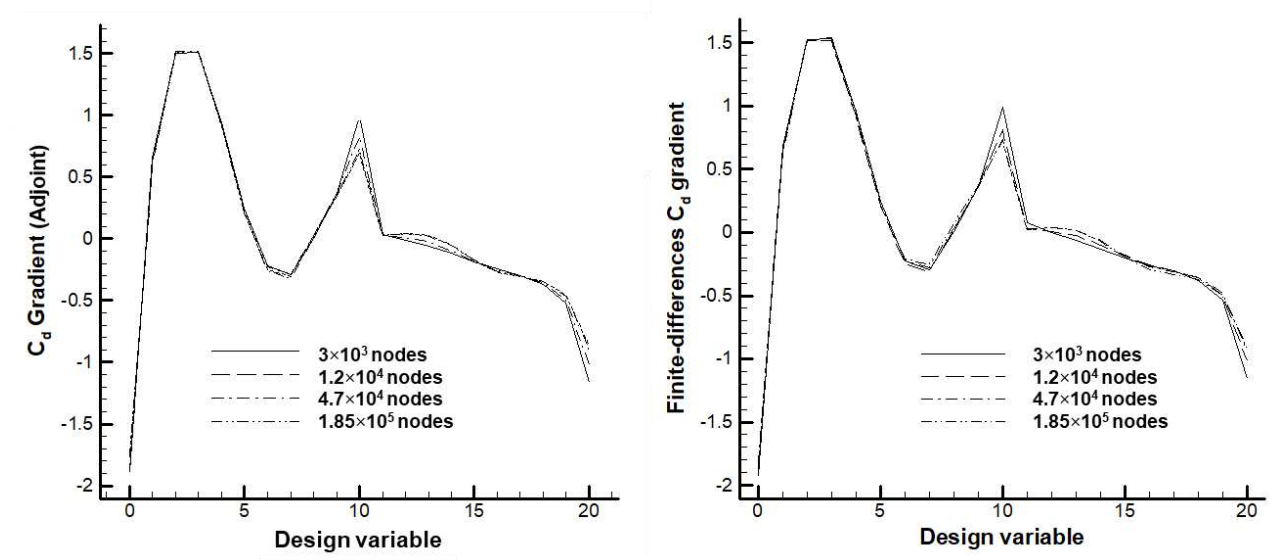

Figure 2. NACA0012 airfoil with $M_{\infty}=0.8$ and $\alpha=1.25^{\circ}$. Adjoint-based (left) and finite-differences drag gradients (right). Design variables correspond to local Hicks-Henne deformation functions.

The first thing to note is that this behavior is not limited to a particular code or scheme: it appears in solutions obtained with continuous and discrete adjoint codes and with different solvers and schemes, including unstructured, cell-vertex codes such as DLR's Tau and Stanford U. code SU2 [5] (see Figure 3), ONERA’s structured, cell-centered ELSA code [6], and others.
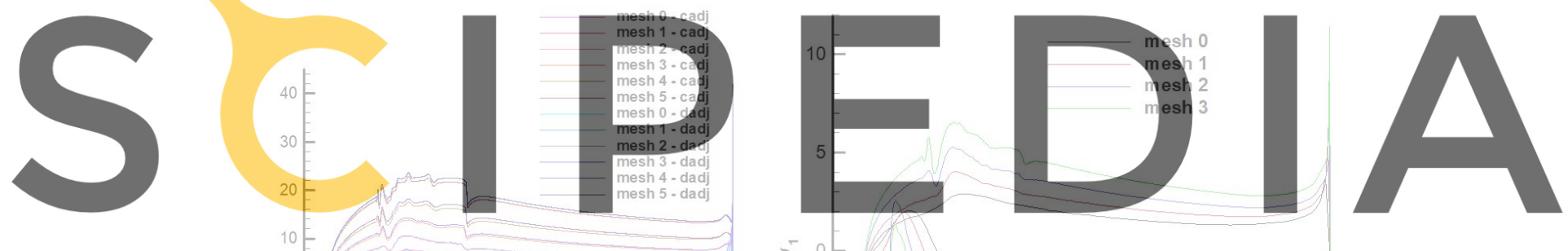

Register for free at https//www.scipedia.com to

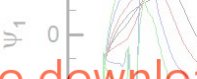

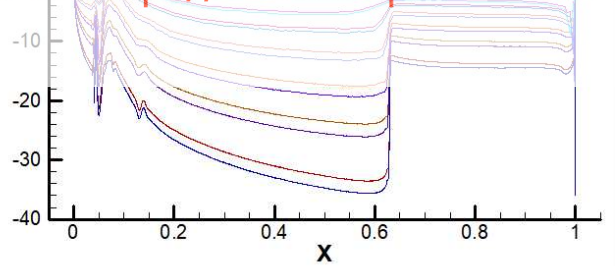

Tau Solver (DLR), Continuous \& Discrete Adj

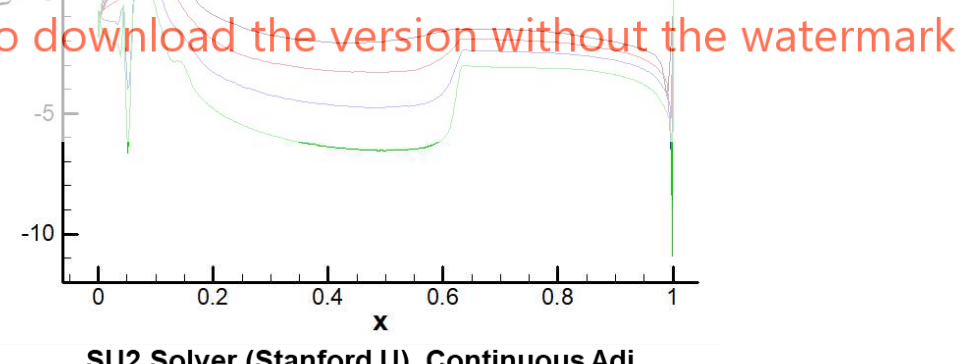

Figure 3. Drag adjoint solution for NACA0012 airfoil at $M_{\infty}=0.8$ and $\alpha=1.25^{\circ}$ with Tau continuous and discrete adjoint solvers and SU2 continuous adjoint solver.

Secondly, the issue also occurs in three dimensional inviscid flows (Figure 4, left), while viscous adjoint solutions, on the other hand, do not appear to be affected (Figure 4, right). 

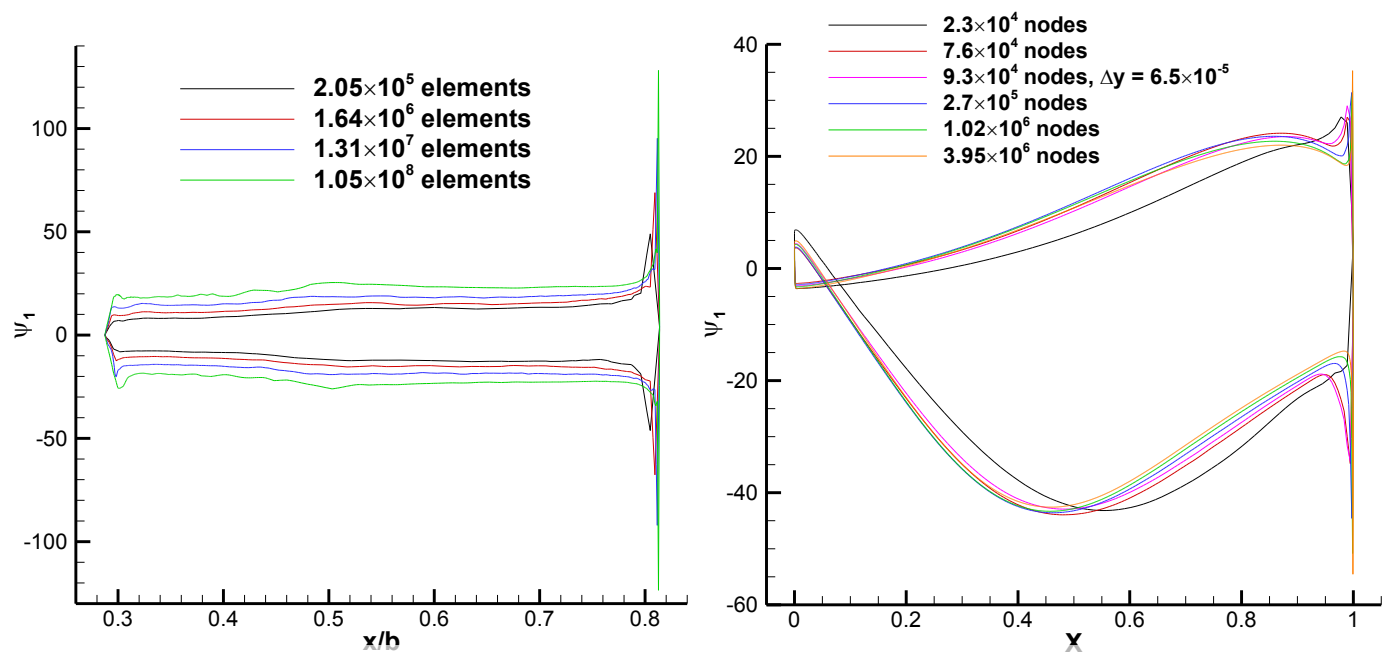

Figure 4. Left: Inviscid lift adjoint solution for an ONERA M6 wing with $M_{\infty}=0.84$ and $\alpha=0^{\circ}$ on 4 mesh levels. Right: Viscous lift adjoint solution for a NACA0012 airfoil at $\operatorname{Re}=5000, M_{\infty}=0.5$ and $\alpha=2^{\circ}$.

\subsection{Dependence on cost function and flow regime}

The issue depends on the cost function and flow regime as follows:

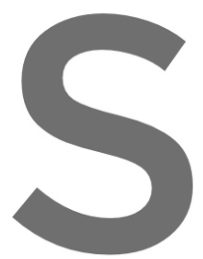

1. drag-based adjoint solutions are only singular (i.e., mesh converging) case singularity is inissing in all cases)

2. Lift-based adjoint solutions show this behavior for an condition (Figure 6), and in all cases the t.e. singularity is

3. Supersonic lift or drag-based adjoint solutions do

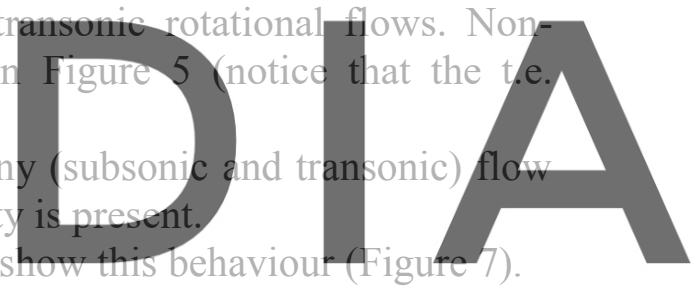

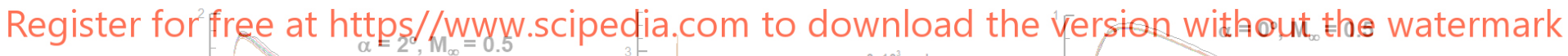
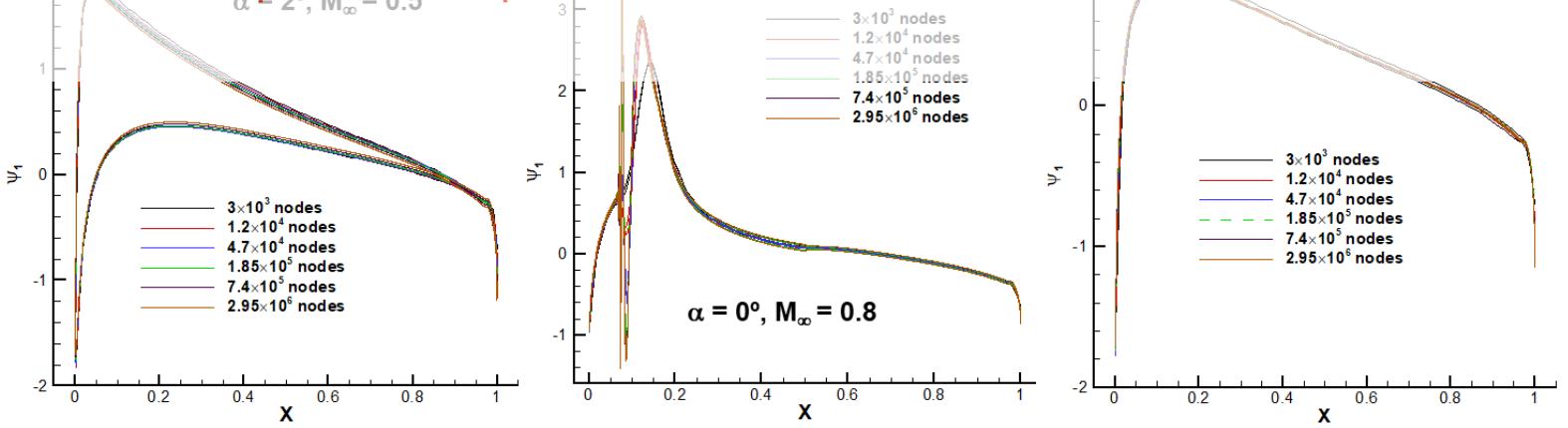

Figure 5. Non-singular drag adjoint solutions for a NACA0012 airfoil 

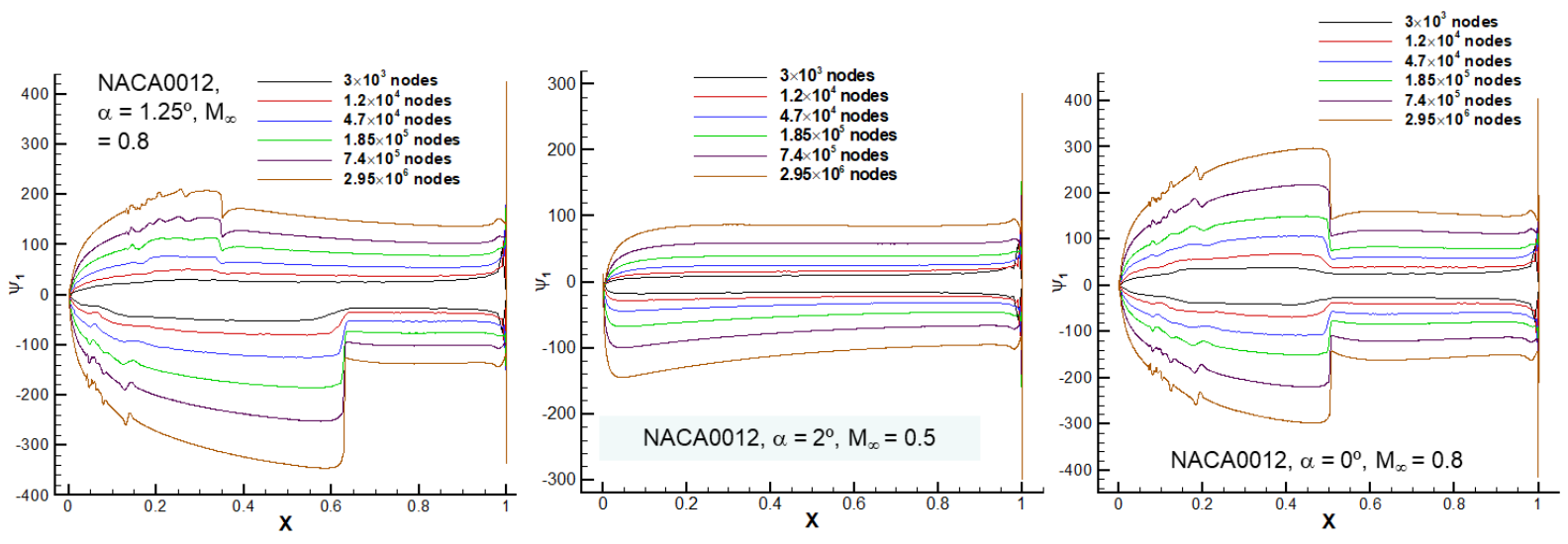

Figure 6. Lift adjoint solutions for a NACA0012 airfoil with different flow conditions.
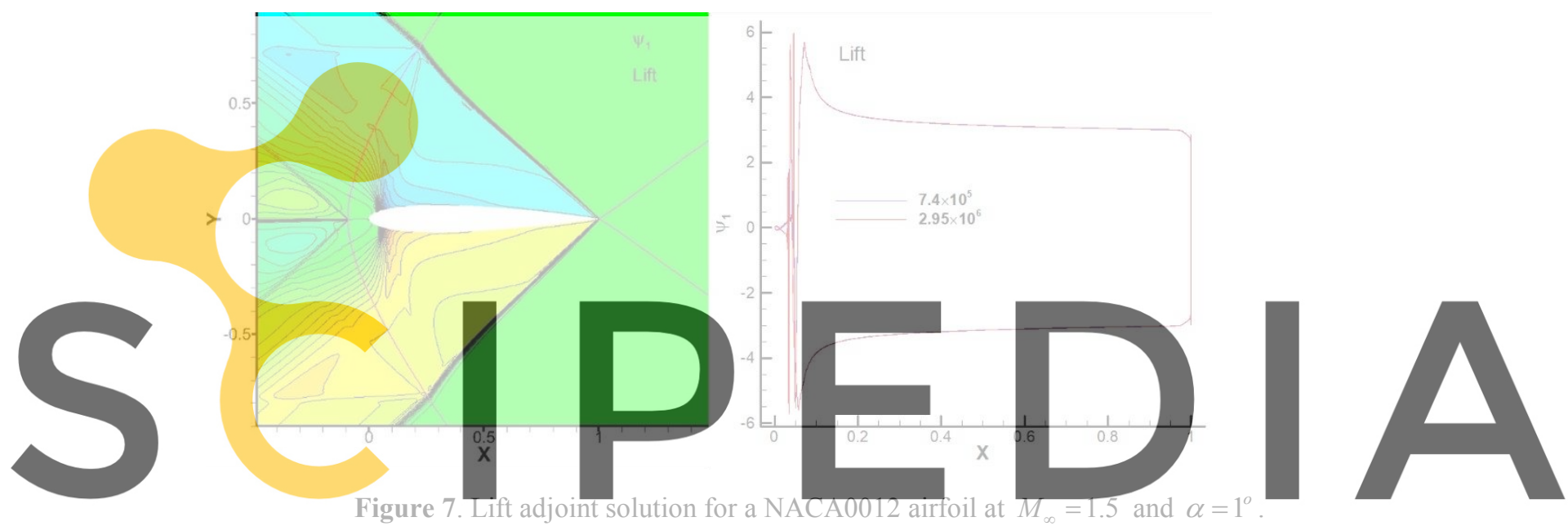

Register for free at https//www.scipedia.com to download the version without the watermark A different cost function $J=\int_{\text {furfeld }} s \vec{v} \cdot \vec{n} d s$, measuring far-field entropy flux, shows the same

behavior as the near-field drag (Figure 8). This is important as the output function is not based on near-field computations and, accordingly, the wall boundary condition is simply $\left(\psi_{x}, \psi_{y}\right) \cdot \vec{n}_{S}=0$ in this case [7].
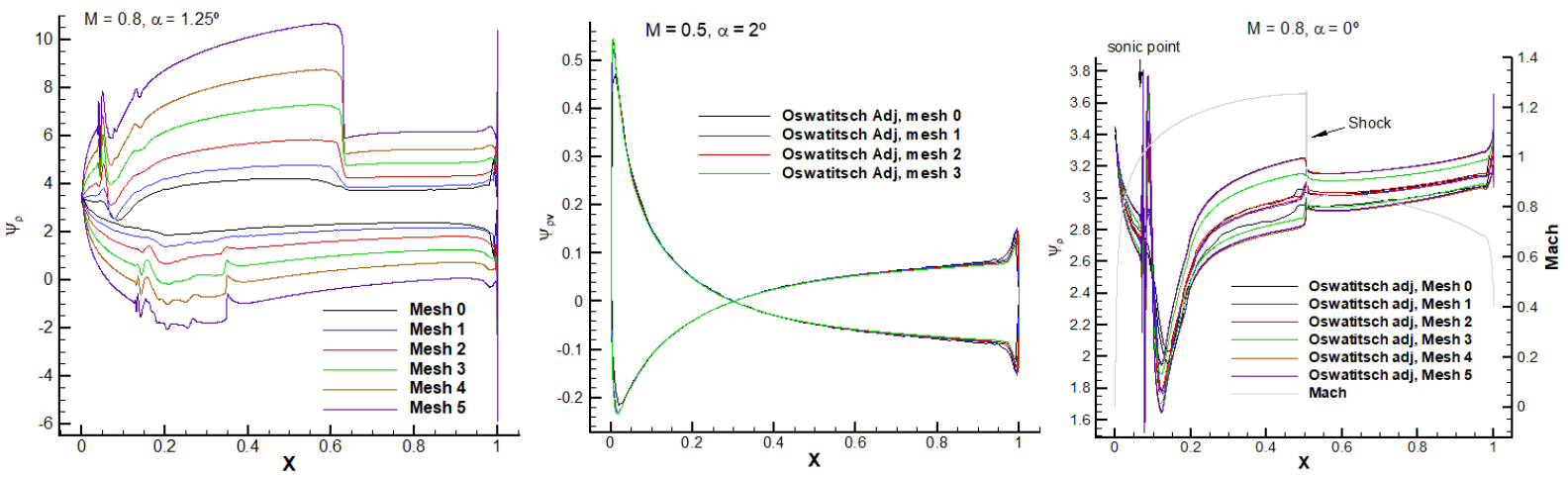

Figure 8. Adjoint of the far-field entropy flux for a NACA0012 airfoil with various flow conditions. 


\subsection{Dependence on far-field and trailing edge geometry}

The problem is rather insensitive to far-field distance and resolution (Figure 9), as well as to the details of the implementation of the adjoint far-field b.c. It is also qualitatively insensitive to the trailing edge geometry (Figure 10).
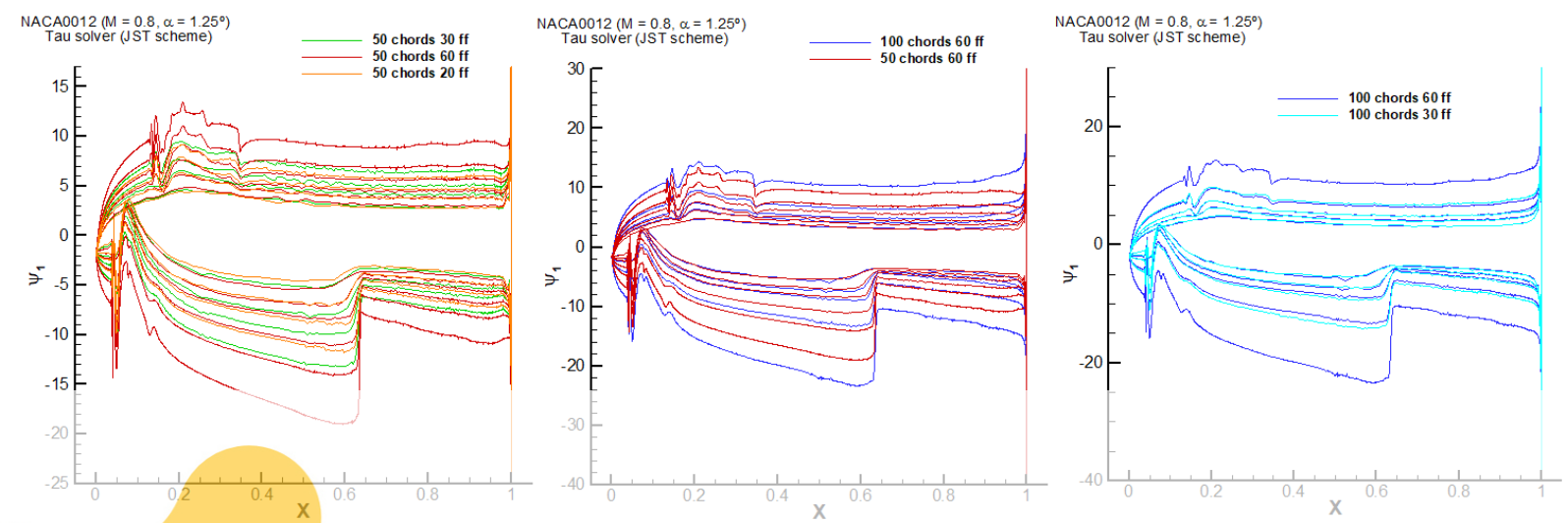

Figure 9. Drag adjoint solution for a NACA0012 airfoil with $M_{\infty}=0.8$ and $\alpha=1.25^{\circ}$. Effect of distance to farfield (in airfoil chords units) and far-field resolution (number of cells in ff).
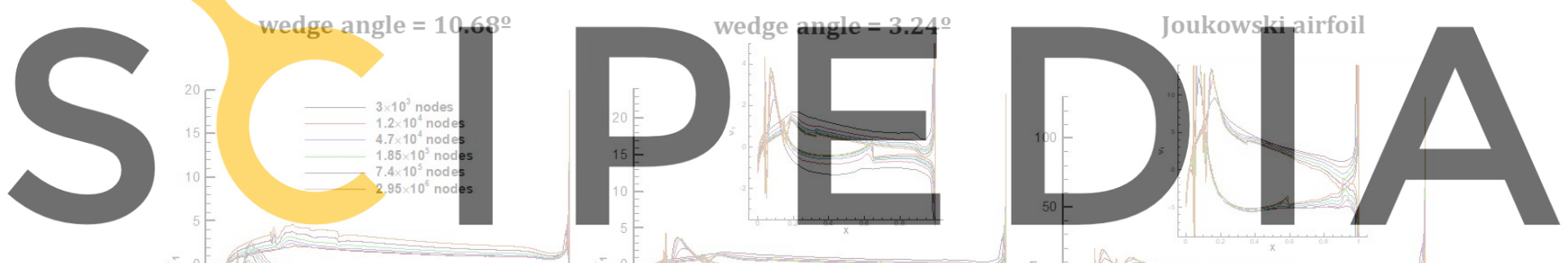

Register for free at https//WwW.scipedia.com to download the version without the watermark
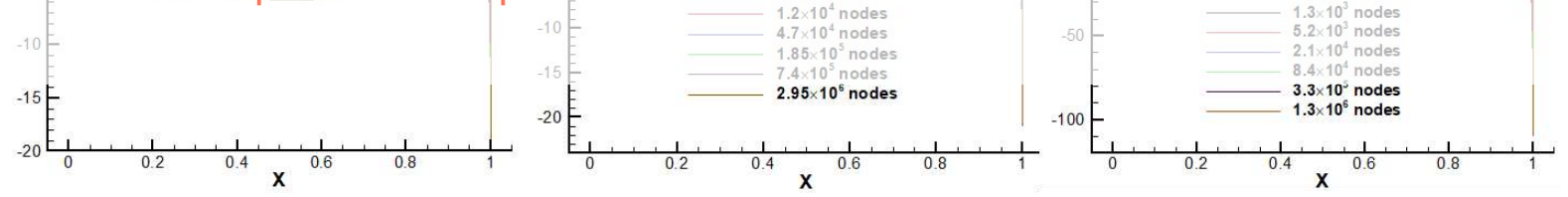

Figure 10. Inviscid drag adjoint solution at with $M_{\infty}=0.8$ and $\alpha=1.25^{\circ}$ for a modified NACA0012 airfoil with progressively reduced wedge angle.

In fact, similar issues are observed in flows past blunt bodies such as the 6:1 ellipse shown in Figure 11. Mimicking the analysis in [2], the adjoint solution in this case shows a $1 / r$ singularity at the rear stagnation point (but not at the rear stagnation streamline) even without a sharp trailing edge. 

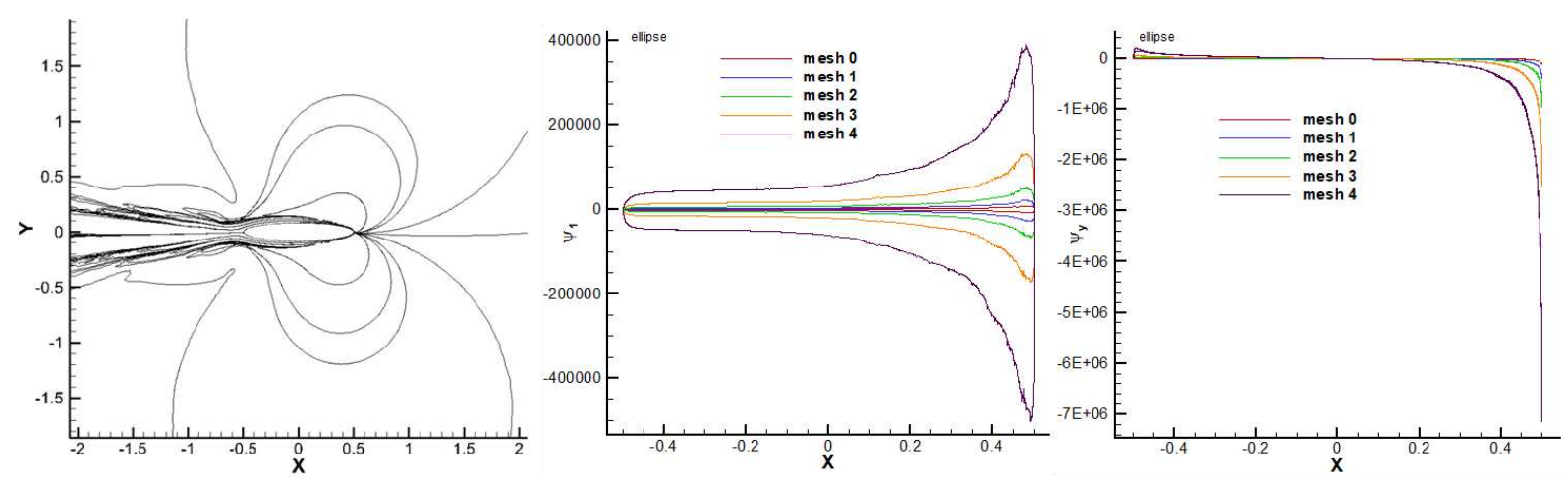

Figure 11. Inviscid lift adjoint solution for a $6: 1$ ellipse with $M_{\infty}=0.2$ and $\alpha=0^{\circ}$.

\subsection{Relation to adioint singularities}

As we have already mentioned, the problem is accompanied by the presence of an adjoint singularity at the trailing edge (Figure 12) but also at the incoming stagnation streamline (Figure 13). In those cases where the adjoint is not singular at those locations, the mesh divergence problem is also absent.
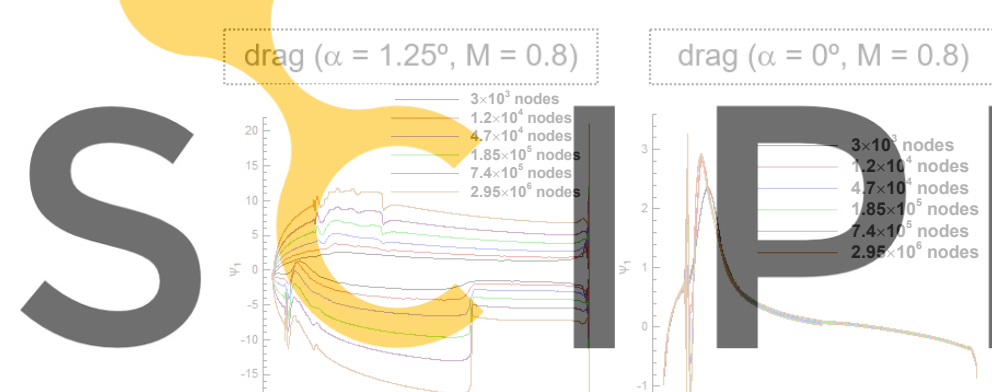

$\operatorname{drag}\left(\alpha=2^{\circ}, M=0.5\right)$

lift $\left(\alpha=2^{\circ}, M=0.5\right)$

Register for free at https//Www.scipedia.com to download the version without the watermark Plots of adjoint "momentum" vector $\left(\psi_{x}, \psi_{y}\right)$
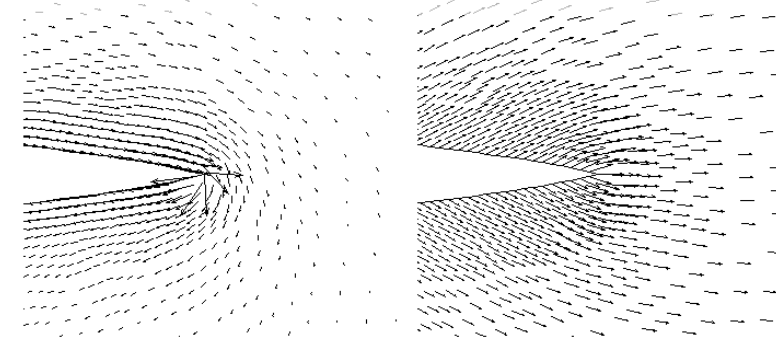

Figure 12. NACA0012: surface adjoint values and plots of adjoint momentum vector $\left(\psi_{x}, \psi_{y}\right)$. 


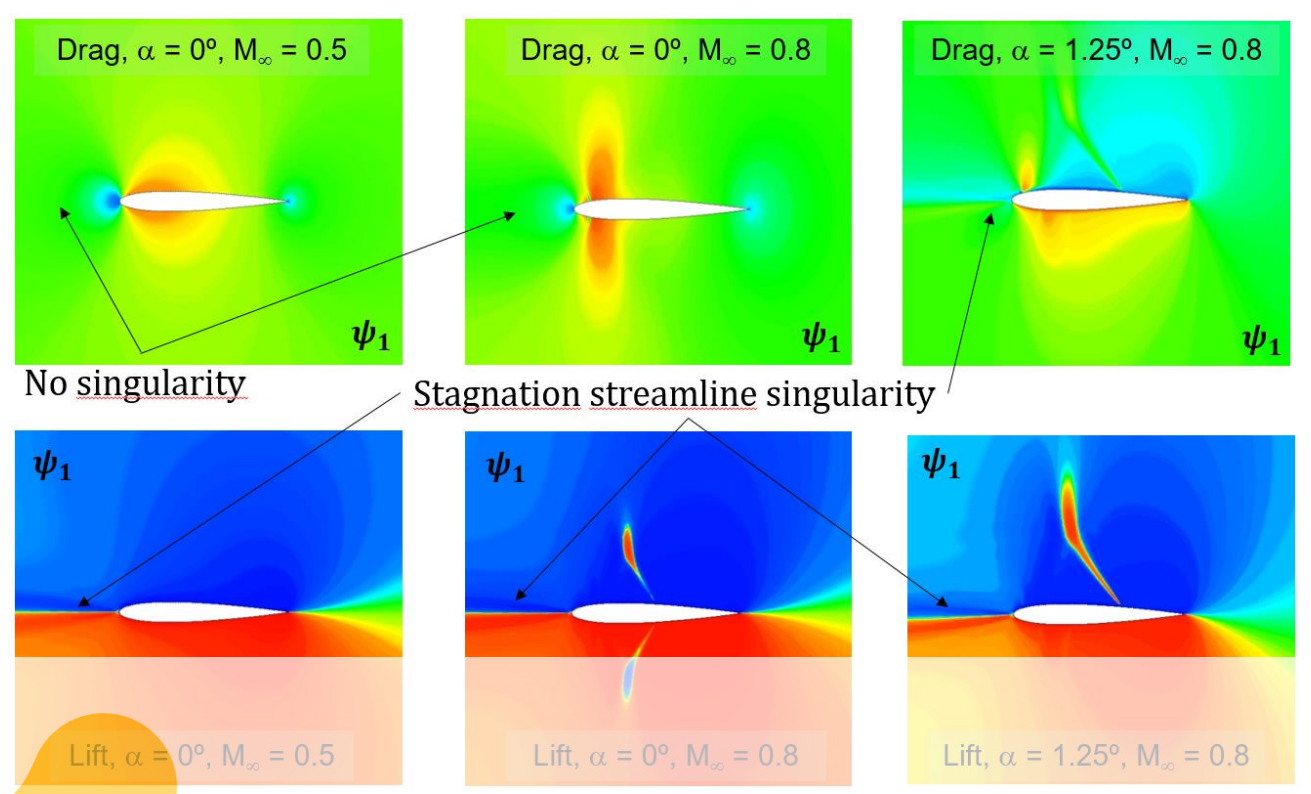

Figure 13. Contour plots of the first component of the lift and drag-based adjoints for a NACA0012 airfoil for several flow conditions.

\subsection{Effect of dissipation}

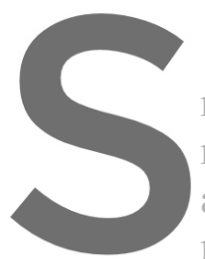

Adjoint solutions for refinement if insufficient dissipation is

require an internal adjo

additional dissipation).

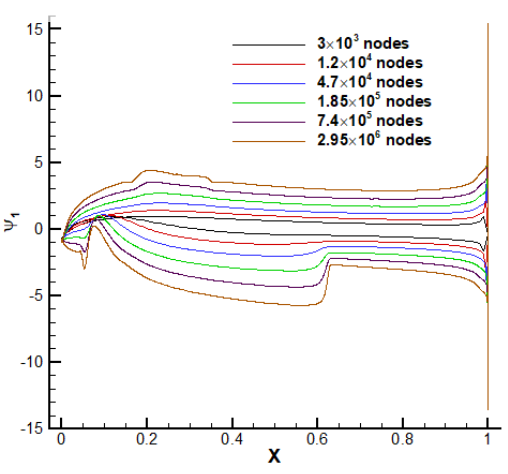

Constant $2^{\text {nd }}$ dissipation (everywhere)

$$
\varepsilon^{(2)}=\text { const }
$$

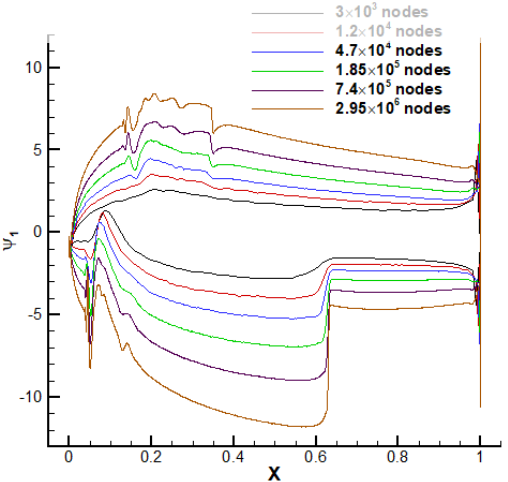

Increasing $2^{\text {nd }}$ dissipation(only at shocks)

$$
\varepsilon^{(2)} \propto 1 / \sqrt{\Delta x}
$$

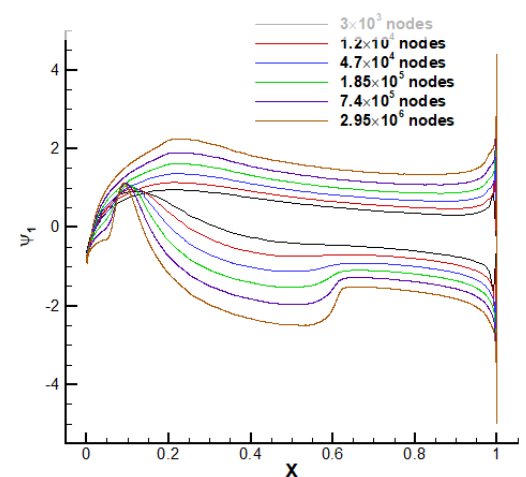

Increasing $2^{\text {nd }}$ dissipation (everywhere)

$$
\varepsilon^{(2)} \propto 1 / \sqrt{\Delta x}
$$


Figure 14. Drag adjoint solution for the NACA0012 airfoil at $M_{\infty}=0.8$ and $\alpha=1.25^{\circ}$ with the JST scheme $d_{i j}=\varepsilon_{i j}^{(2)}\left(U_{i}-U_{j}\right)+\varepsilon_{i j}^{(4)}\left(\sum_{k: i}\left(U_{k}-U_{i}\right)-\sum_{k: j}\left(U_{k}-U_{j}\right)\right)$ and modified $2^{\text {nd }}$ dissipation coefficient $\varepsilon^{(2)}$.

On the other hand, the actual value of the adjoint solution at the wall has been seen to depend on the dissipation level. Focusing on a subcritical case, where it is safe to set $\varepsilon^{(2)}=0$, it turns out that, on a given mesh, the adjoint solution depends strongly on the dissipation level, in such a way that reducing the dissipation actually mimics the effect of mesh refinement (Figure 15)

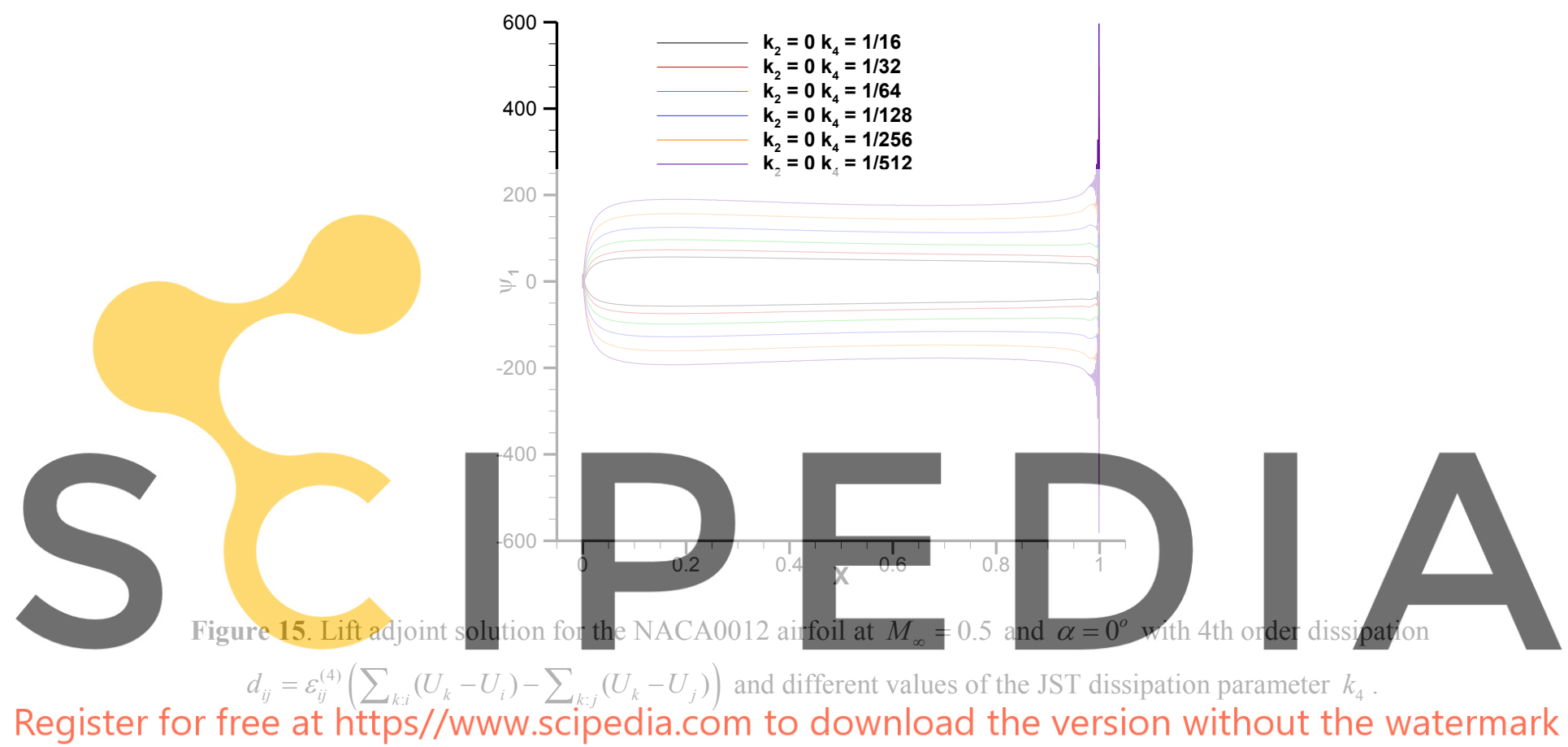

\section{POINT SOURCE PERTURBATIONS}

Green's functions are closely related to adjoint equations. If $G$ is a Green's function obeying the linearized Euler equations $\nabla \cdot\left(\vec{F}_{U} G(\vec{x}, \vec{y})\right)=f(\vec{y}) \delta(\vec{x}-\vec{y})$, then its effect $\delta J(\vec{y})=J(U+G)-J(U)$ on a cost function $J$ can be alternatively computed as $\delta J(\vec{y})=\psi^{T}(\vec{y}) f(\vec{y})$ where $\psi$ is the adjoint solution corresponding to $J$. Choosing the source vectors $f$ in a clever way (such that the resulting $\delta J$ is easy to compute), it is possible to obtain information about the adjoint solutions. This program was completed with a closed-form solution for the adjoint quasi-1D Euler equations in [9] and initiated for the 2D Euler equations in [2], where the following four linearly independent source vectors representing mass, normal force, enthalpy and total pressure perturbations, respectively, were considered 


$$
\begin{aligned}
& \delta J_{1}=\psi_{1}+\vec{v} \cdot\left(\psi_{x}, \psi_{y}\right)+H \psi_{4} \\
& \delta J_{2}=-\rho v \psi_{x}+\rho u \psi_{y} \\
& \delta J_{3}=-\frac{1}{2 H} \psi_{1}+\frac{1}{2} \psi_{4} \\
& \delta J_{4}=\frac{1}{p_{0}}\left(\frac{\gamma}{\gamma-1}+\frac{1}{\gamma M^{2}}\right) \delta J_{1}+\frac{1}{p_{0}} \frac{1}{\gamma M^{2}} \delta J_{5}
\end{aligned}
$$

(here $\delta J_{5}=\vec{v} \cdot\left(\psi_{x}, \psi_{y}\right)$ ). The above choices are interesting because $\delta J_{1}$ is related to the (continuous) adjoint-based lift/drag gradient (see for example [10])

$$
\delta \int_{S} C_{p}(\vec{n} \cdot \vec{d}) d s=\int_{\delta S} C_{p}(\vec{n} \cdot \vec{d}) d s-\int_{S}(\vec{n} \cdot \delta \vec{v}) \rho \delta J_{1} d s
$$

( $S$ denotes the wall surface, and the first part contains the geometric variation of the objective function); $\delta J_{2}$ approaches $\rho q \vec{n}_{S} \cdot\left(\psi_{x}, \psi_{y}\right)$ as the insertion point approaches the wall, where $\vec{n}_{S} \cdot\left(\psi_{x}, \psi_{y}\right)$ is fixed by the adjoint b.c.; $\delta J_{3}$ is identically zero for pressure-based cost functions such as lift or drag; finally, $\delta J_{4}$ was conjectured in [2] to lead to a non-uniform mass perturbation along the streamline downstream the insertion point that would be responsible for the adjoint singularity along the incoming stagnation streamline.

It has been recently pointed out [6] that computing $\delta J_{i}$ with a numerical solution can shed light on the structure of adjoint solutions and, particularly, on the problem at hand. $\delta J_{i}$ can be

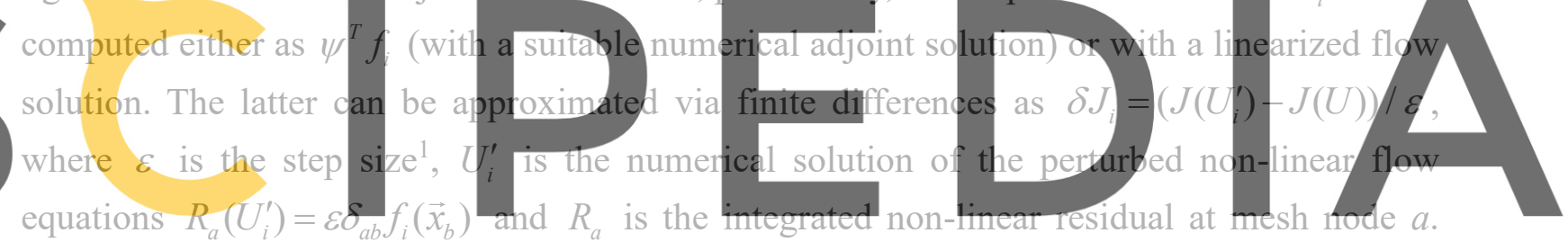

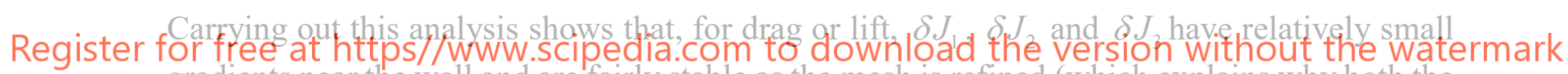
gradients near the wall and are fairly stable as the mesh is refined (which explains why both the sensitivity derivatives and the adjoint b.c. behave well with mesh refinement), while $\delta J_{4}$ grows strongly towards the wall (see Figure 16). Actually, for a given point, mesh refinement does not change much the value, but as finer meshes are considered the perturbation point can be placed closer to the wall. The latter behavior is also shared by $\delta J_{5}$, which would correspond to a force applied in the local flow direction. Further experimentation (not shown) indicates that placing the perturbation point directly at a wall node yields a finite value for both $\delta J_{4}$ and $\delta J_{5}$ which grows as the mesh is refined.

\footnotetext{
${ }^{1}$ The value of the step size is taken as $\varepsilon=10^{-9}$ in all cases. Results obviously depend on the choice of step size, which was not optimized for accuracy for all perturbations, although a reasonable effort was made to determine an acceptable value by scanning a large range of values $10^{-4}-10^{-10}$ and choosing the step size that did not significantly change the results.
} 

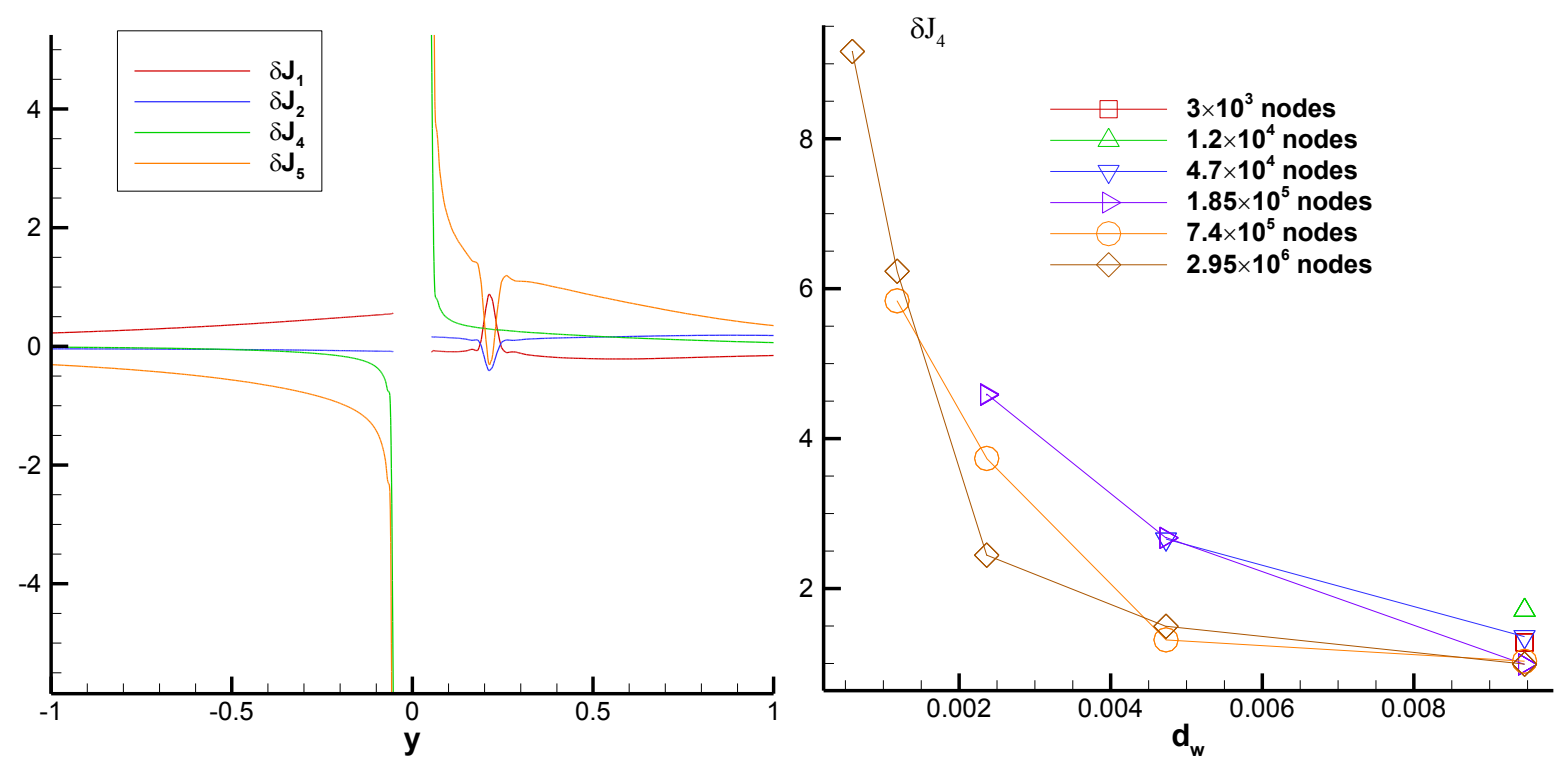

Figure 16. NACA0012 with $M_{\infty}=0.8$ and $\alpha=1.25^{\circ}$. Left: plot of $\delta J_{i}$ for drag on mesh $3\left(1.85 \times 10^{5}\right.$ nodes $)$ computed as $\psi^{T} f_{i}$ along a vertical line at $x / c=0.5$. Right: $\delta J_{4}$ computed via finite-differences on 6 sequentially refined meshes at selected points along the line $x / c=0.5$.

\section{SUMMARY AND DISCUSSION}

We have shown that 2D and 3D inviscid continuous and discrete adjoint solutions are generically strongly mesh dependent at and near walls and do not converge as the mesh is refined. Lift-based adjoint solutions are affected for any flow condition (subsonic or transonic), while drag-based adjoint solutions are affected for transonic lifting flows. As this issue has been observed in totally unrelated solvers with different schemes, we assume that the mesh dependence is not caused by a bug or an inconsistency of the adjoint scheme.

Mesh dependence of adjoint solutions has been observed for shocked flows and can be cured with carefully tuned levels of dissipation at the shock [8], but the problem we are dealing with here also appears in non-shocked flows and is largely insensitive to dissipation levels at the shock region. A certain amount of (local) mesh dependence can also be expected near adjoint singularities (the trailing edge, the shock foot, the supersonic characteristic, the stagnation streamline, the entrance to the supersonic zone along the wall, etc), but in our case the mesh dependence extends to all the wall boundary. The fact that it is correlated with the trailing edge singularity led us at first to conjecture that the problem is a numerical effect caused by the t.e. singularity. However, recent evidence ([6] and Figure 16) shows that there might actually be an adjoint singularity along the wall (with the same origin as the one along the incoming stagnation streamline). The presence of a singularity at the wall would certainly explain the behavior (it appears that, without dissipation, the adjoint values would grow unbounded), but it would remain to determine if the singularity is of numerical or analytic origin and, in the latter case, how a singular (i.e. infinite) adjoint solution could be reconciled with the adjoint wall b.c. For the numerical solution, $\psi_{1}, \psi_{x}, \psi_{y}, \psi_{4}$ appear to diverge while the quantities $\delta J_{1}=\psi_{1}+\vec{v} \cdot\left(\psi_{x}, \psi_{y}\right)+H \psi_{4}, \delta J_{2}=-\rho v \psi_{x}+\rho u \psi_{y}$ and $\delta J_{3}=-\frac{1}{2 H} \psi_{1}+\frac{1}{2} \psi_{4}$ remain finite, so 
this could offer a hint of the mechanism at work in the case that there is an analytic singularity at the wall. Such singularity was not found, though not completely excluded either, in [2] by the analysis of adjoint solutions using the Green's function approach. He have analyzed $\delta J_{4}$ near the wall as was done in [2] for the stagnation streamline, but this approach has not yielded any useful result so far, neither in the simple plane stagnation-point potential flow approximation considered in [2], nor in a more complex case involving potential flow around a circular cylinder. In both cases, $\delta J_{4}$ tends to zero as the insertion point approaches the wall. However, our analysis is based on the simple linearized solution

$$
u_{4}(\vec{x}, \vec{\xi})=\mathcal{H}(s) \delta(n)(\rho q)^{-1}\left(\partial U / \partial p_{0}\right)_{\alpha, p, H}-\int_{0}^{\infty}(d \tilde{m} / d s) u_{1}\left(\vec{x}, \vec{x}^{\prime}\left(s^{\prime}, 0\right)\right) d s^{\prime}
$$

derived in [2], which is incomplete (it does not obey the linearized equations $\left.\nabla \cdot\left(\vec{F}_{U} u_{4}(\vec{x}, \vec{\xi})\right)=f_{4}(\vec{\xi}) \delta(\vec{x}-\vec{\xi})\right)$, and the complete solution is far more complex to analyze.

We intend to address these issues in future work.

\section{REFERENCES}

[1] Lozano, C. Watch Your Adjoints! Lack of Mesh Convergence in Inviscid Adjoint Solutions. AIAA J. (2019) 57(9):3991-4006.

[2] Giles, M. and Pierce, N. Adjoint equations in CFD: Duality, boundary conditions and solution behavior. AIAA paper 97-1850 (1997).

[3] Giles, M. and Pierce, N. Improved lift and drag estimates using adjoint Euler equations. AIAA paper 99-3293 (1999).

[4] Schwamborn, D., Gerhold, T. and Heinrich, R. The DLR TAU-code: Recent applications in research and industry. Proceedings of ECCOMAS CFD 2006.

[5] Economon, T., Palacios, F., Copeland, S., Lukaczyk, T. and Alonso, J. SU2: An OpenSource Suite for Multiphysics Simulation and Design. AIAA J. (2016) 54(3):828-846.

[6] Peter, J., Renac, F. and Labbé, C. Analysis of finite-volume discrete adjoint fields for 2D compressible Euler flows. Preprint (2020). arXiv:2009.07096 [physics.comp-ph].

[7] Lozano, C. Entropy and Adjoint Methods. J. Sci. Comput. (2019) 81:2447-2483.

[8] Giles, M. Discrete adjoint approximations with shocks. In: Hou T.Y., Tadmor E. (eds) Hyperbolic Problems: Theory, Numerics, Applications, pp 185-194 (2003). Springer.

[9] Giles, M. and Pierce, N. Analytic adjoint solutions for the quasi-one-dimensional Euler equations. J. Fluid Mech. (2001) 426: 327-345.

[10] Castro, C., Lozano, C., Palacios, F. and Zuazua, E. Systematic continuous adjoint approach to viscous aerodynamic design on unstructured meshes. AIAA J. (2007) 45(9):2125-2139. 